\title{
Selective and flexible depletion of problematic sequences from RNA-seq libraries at the CDNA stage
}

\author{
Stuart K Archer ${ }^{1}$, Nikolay E Shirokikh² and Thomas Preiss ${ }^{1 *}$
}

\begin{abstract}
Background: A major hurdle to transcriptome profiling by deep-sequencing technologies is that abundant transcripts, such as rRNAs, can overwhelm the libraries, severely reducing transcriptome-wide coverage. Methods for depletion of such unwanted sequences typically require treatment of RNA samples prior to library preparation, are costly and not suited to unusual species and applications. Here we describe Probe-Directed Degradation (PDD), an approach that employs hybridisation to DNA oligonucleotides at the single-stranded cDNA library stage and digestion with Duplex-Specific Nuclease (DSN).

Results: Targeting Saccharomyces cerevisiae rRNA sequences in Illumina HiSeq libraries generated by the split adapter method we show that PDD results in efficient removal of rRNA. The probes generate extended zones of depletion as a function of library insert size and the requirements for DSN cleavage. Using intact total RNA as starting material, probes can be spaced at the minimum anticipated library size minus 20 nucleotides to achieve continuous depletion. No off-target bias is detectable when comparing PDD-treated with untreated libraries. We further provide a bioinformatics tool to design suitable PDD probe sets.

Conclusion: We find that PDD is a rapid procedure that results in effective and specific depletion of unwanted sequences from deep-sequencing libraries. Because PDD acts at the cDNA stage, handling of fragile RNA samples can be minimised and it should further be feasible to remediate existing libraries. Importantly, PDD preserves the original RNA fragment boundaries as is required for nucleotide-resolution footprinting or base-cleavage studies. Finally, as PDD utilises unmodified DNA oligonucleotides it can provide a low-cost option for large-scale projects, or be flexibly customised to suit different depletion targets, sample types and organisms.
\end{abstract}

Keywords: RNA-seq, rRNA, Library, cDNA, Duplex specific nuclease, Depletion, PDD

\section{Background}

The application of RNA-seq for in-depth transcriptomics analyses is hindered by the vast excess of certain RNAs leading to insufficient coverage of transcripts of interest. Such problematic sequences can be rRNA (or fragments thereof, e.g. as in degraded archival samples), RNAs from particular species (e.g. in environmental or host-pathogen transcriptomics) or canonical transcript sequences (e.g. in targeted sequencing to identify rare variants/modifications [1]). A particular challenge for experiments involving

\footnotetext{
* Correspondence: thomas.preiss@anu.edu.au

'Genome Biology Department, The John Curtin School of Medical Research (JCSMR), The Australian National University, Acton, Canberra, Australian Capital Territory, Australia

Full list of author information is available at the end of the article
}

targeted isolation of complex-associated RNA, such as in ribosome profiling [2,3], CLIP-seq [4,5], or modified base cleavage studies is that the RNA must be partially fragmented, which releases a plethora of rRNA digestion products. This impedes interpretation of the results; for example in CLIP-seq, an input sample digested in parallel should be sequenced as a control to facilitate peak-calling from the sequence data [6], however this is rarely done in practice due to the high content of degraded rRNA in these controls.

Popular methods to deplete unwanted sequences involve hybridizing the RNA with biotinylated LNA probes (as in several commercial kits) or hybridizing the single-stranded cDNA library to biotinylated DNA probes [3], and depleting the bound targets using immobilized streptavidin. 
However, these probes are usually purchased as readymade sets targeted at rRNA in a limited range of source species, precluding customization. The costs of obtaining custom modified probes to, for instance, target all rRNA fragments in degraded (e.g. archival samples) or digested samples (e.g. for CLIP-seq or ribosome profiling) are prohibitive. Targeting rRNA with unmodified antisense DNA oligonucleotides and digestion with RNase $\mathrm{H}$ can yield efficient depletion without introducing gross bias into the transcriptome $[7,8]$. This approach, however, requires saturation of rRNA with contiguous oligonucleotides. Further, due to the high thermal stability of RNA:DNA hybrids [9] and the short duplex length (six base pairs) targeted by RNase $\mathrm{H}$, there is no guarantee that the resulting fragment ends will all be faithful representations of the input fragment ends in nucleotide-resolution studies (particularly as RNase $\mathrm{H}$ yields polished, easily ligated RNA ends).

A strategy to deplete high-abundance sequences from dsDNA libraries is $\mathrm{C}_{0} \mathrm{~T}$-hybridization [10], which involves heat-denaturing followed by re-annealing. High-abundance sequences, which preferentially re-anneal, are removed using duplex-specific nuclease (DSN) [11] or hydroxyapatite chromatography [12]. This procedure has not been widely adopted due to the difficulty of fine-tuning the extended hybridization reaction. Application of $\mathrm{C}_{0} \mathrm{~T}$-hybridization to deep sequencing libraries is further severely limited by the potential of the ubiquitous linker/adapter sequences to be inadvertently targeted. Notably, DSN treatment in conjunction with targeted DNA oligonucleotide probes has been used to deplete abundant mRNA sequences in conventional, full-length cDNA libraries [13].

Here we outline probe-directed degradation (PDD), a DSN/probe hybridization-based method for depletion of unwanted cDNA sequences from RNA-seq libraries. Targeting Saccharomyces cerevisiae rRNA we achieved efficient depletion across multiple PDD-targeted loci without introducing bias among mRNA-derived sequences. We present probe design guidelines and a bioinformatics tool that evaluates the efficacy of candidate probe sets. We also describe some streamlining modifications to the split adapter library preparation strategy. Overall, we find PDD to be rapid and specific, while also being quickly and cheaply customizable to diverse species or applications.

\section{Methods}

\section{Probe design}

Probes were initially designed manually to target rRNA sequences that form the major contaminants in ribosome profiling libraries (data not shown). To enable in silico analysis of these probes for possible hybridization with other transcripts, we constructed a Perl script to identify matches of $>10 \mathrm{nt}$ in the transcribed portion of any annotated genome (in GenBank or Ensembl format), estimating the $\mathrm{T}_{\mathrm{m}}$ of each target and off-target match by the method of Allawi et al. [14] as implemented in the BioPerl Primer module. Here we used the reference genome for S. cerevisiae S288C, assembly R64-1-1 (Saccharomyces Genome Database [15]). Probe sequences (Additional file 1: Table S1) were refined in several iterations to maximize the $\mathrm{T}_{\mathrm{m}}$ differential between desired and undesired targets. They were then ordered in plate format from Integrated DNA Technologies and individually resuspended at $200 \mu \mathrm{M}$.

\section{Library preparation}

A "degraded" RNA sample (used in qPCR analysis) was generated by incubating $1 \mathrm{AU}_{260}$ of $S$. cerevisiae cytoplasmic lysate with $3 \mathrm{U}$ of RNase 1 for 30 minutes at room temperature. A "total" RNA library (used for sequencing) was generated by $\mathrm{Mg}^{+2}$-mediated cleavage of intact RNA for 8 minutes at $94^{\circ} \mathrm{C}$ using the NEBNext $\mathrm{Mg}^{+2}$ RNA fragmentation module (New England Biolabs). A "RiboMinus ${ }^{\mathrm{Tm}}$ spike-in" library (for assessing mRNA coverage and PCR duplication, was generated by mixing RiboMinus ${ }^{\text {TM }}$-treated RNA (Life Technologies, generated as per manufacturer's instructions) with total RNA in a 1:4 ratio and fragmenting for 6 minutes at $94^{\circ} \mathrm{C}$. In both cases, libraries were generated by the split adapter method as described [2] with modifications (ExoI digestion and SPRI bead selection to rid the library of unextended RT primer, see below).

Library preparation was performed as follows. After ethanol precipitation and resuspension in $\mathrm{T}_{10} \mathrm{E}_{0.1},(10 \mathrm{mM}$ Tris, $0.1 \mathrm{mM}$ EDTA buffer $\mathrm{pH}$ 7.4) approximately $500 \mathrm{ng}$ of fragmented RNA was end-repaired by $5 \mathrm{U}$ of T4 Polynucleotide Kinase (wild-type, New England Biolabs) in $20 \mu \mathrm{l}$ of $1 \times$ PNK buffer (no ATP) for 2 hours at $37^{\circ} \mathrm{C}$ in the presence of $1 \mathrm{U} / \mu \mathrm{l}$ of RNaseOUT inhibitor (Life Technologies). T4 PNK was then heat-inactivated at $65^{\circ} \mathrm{C}$ for 10 minutes and the resultant RNA was $3^{\prime}$ polyadenylated using the Ambion Poly(A)-tailing kit as follows. The PNK reaction was added to a master mix to give a final $50 \mu \mathrm{l}$ reaction containing of $0.5 \mathrm{mM}$ rATP, $2.5 \mathrm{mM} \mathrm{MnCl}_{2}, 1 \times$ reaction buffer, $0.75 \mathrm{U}$ of E. coli Poly(A) Polymerase and supplemented with fresh RNaseOUT RNAse inhibitor (Life Technologies). The polyadenylation reaction was allowed to proceed for 1 hour at $37^{\circ} \mathrm{C}$. Pyrophosphate precipitate was pelleted by brief centrifugation and the RNA in the supernatant was ethanol-precipitated in a new tube. RNA pellets were resuspended in $15 \mu \mathrm{l}$ of $1 \mathrm{mM}$ sodium citrate buffer, $\mathrm{pH}$ 6.2. After polyadenylation, $\mathrm{A}_{260}$ readings were no longer useful for quantifying RNA.

Half of the resuspended RNA ( $7 \mu$ l, about $250 \mathrm{ng}$ of the original RNA) was reverse transcribed using the SuperScript ${ }^{\circ}$ III first-strand synthesis kit (Life Technologies) according to the manufacturer's instructions with some variations as follows. 20 pmol of split adapter/oligo dT primer (Integrated DNA Technologies) were mixed with the 
template RNA and dNTPs in $13 \mu \mathrm{l}$ and heated to $70^{\circ} \mathrm{C}$ for 3 minutes in a thermocycler, cooled to $60^{\circ} \mathrm{C}$ then slow-ramped to $55^{\circ} \mathrm{C}$ over 2 minutes to anneal primer. A preheated $7 \mu \mathrm{l}$ reverse transcription master mix aliquot (containing buffer, SuperScript ${ }^{\circ}$ III and RNaseOUT $^{\mathrm{rm}}$ ) was added, the mixture was slow-ramped to $50^{\circ} \mathrm{C}$ and incubated for a further 30 minutes. The reverse transcription reaction was heated to $60^{\circ} \mathrm{C}$ for 5 minutes, then cooled to $37^{\circ} \mathrm{C}$, and $1 \mu \mathrm{l}$ of $20 \mathrm{U} / \mu \mathrm{l}$ ExoI (New England Biolabs) was immediately added, followed by a further 20 minute incubation to allow depletion of the unextended single-stranded primer. Meanwhile, AMPure XP beads (Beckman Coulter) from $20 \mu \mathrm{l}$ of initial manufacturer's suspension were washed and resuspended in $2.2 \times$ reaction volumes $(44 \mu \mathrm{l})$ of $1 \times \mathrm{PN}$ buffer (20\% PEG-8000, $2.5 \mathrm{M} \mathrm{NaCl}$ ). ExoI was inactivated by adding $1 \mu \mathrm{l} 250 \mathrm{mM}$ EDTA, and both the reaction mixture $(22 \mu \mathrm{l})$ and the beads suspension $(44 \mu \mathrm{l})$ were preheated in a $60^{\circ} \mathrm{C}$ oven before rapidly mixing and returning to the oven for 5 minutes. Beads were collected on a magnetic rack for 1 minute in the oven, the rack was transferred to room temperature and the supernatant was immediately removed and replaced with roomtemperature $0.66 \times \mathrm{PN}$ buffer while keeping the reaction tubes on the magnet. After two 230 second washes in $70 \%$ ethanol on the magnet at room temperature, beads were dried $\sim 2$ minutes and cDNA was eluted in $13.3 \mu \mathrm{l}$ $\mathrm{T}_{10} \mathrm{E}_{0.1}$ buffer. The purpose of the elevated temperature during bead binding was to prevent non-specific primer annealing and carryover, while still leaving cDNA:RNA duplexes intact to take advantage of the large length differential between the polyadenylated RNA and the primer for size-based selection on Ampure XP beads. We have not tested room-temperature separation conditions.

\section{CDNA circularization}

Purified cDNA:RNA duplexes from the previous step were denatured at $80^{\circ} \mathrm{C}$ for 15 minutes and the RNA degraded by adding $0.7 \mu \mathrm{g}$ of RNase A and incubating at $37^{\circ} \mathrm{C}$ for 20 minutes. An aliquot $(9 \mu \mathrm{l})$ was circularised with $75 \mathrm{U}$ of CircLigase ${ }^{\mathrm{Tn}}$ II (Epicentre) in a $20 \mu \mathrm{l}$ selfligation reaction containing $1 \times$ manufacturer's reaction buffer supplemented with $2.5 \mathrm{mM} \mathrm{MnCl} 2$ and $0.5 \mathrm{M}$ betaine for 2-6 hours at $60^{\circ} \mathrm{C}$. Upon completion of the circularisation reaction, EDTA was added to $2.5 \mathrm{mM}$ and the ligase was heat-inactivated at $80^{\circ} \mathrm{C}$ for 15 minutes. The amount of cDNA product in the reaction was that derived from approximately 112 ng of starting RNA (however, we have successfully used as little as $90 \mathrm{ng}$ in a $20 \mu \mathrm{l}$ circularisation reaction). Circular cDNA was then purified using AMPure XP beads (using $20 \mu \mathrm{l}$ of the initial bead mixture, washed and resuspended in $2 \times$ sample volumes of $\mathrm{PN}$ buffer at ambient temperature, and proceeding with binding and washing as per the manufacturer's instructions) and the resultant bound cDNA library was eluted from the beads in $10 \mu \mathrm{l} \mathrm{T}_{10} \mathrm{E}_{0.1}$ buffer.

\section{DD treatment}

Half of the circularised library $(5 \mu \mathrm{l})$ was mixed with $2 \mu \mathrm{l}$ of $3.5 \times$ DSN buffer (Evrogen) with depletion probes (50 probes at $0.81 \mu \mathrm{M}$ each) so that each probe was approximately equimolar with the library assuming $100 \%$ conversion of RNA to cDNA. The $7 \mu \mathrm{lmix}$ was overlayed with mineral oil and denatured on a thermocycler at $95^{\circ} \mathrm{C}$ for 1 minute, brought to $75^{\circ} \mathrm{C}$ and then slowly cooled $\left(3^{\circ} \mathrm{C} /\right.$ minute $)$ to $48^{\circ} \mathrm{C}$. After a 5 minute hybridization at $48^{\circ} \mathrm{C}, 3 \mu \mathrm{l}$ of pre-warmed DSN master mix containing 0.4 U DSN (Evrogen) in 1× DSN buffer was added and incubated for 20 minutes. The reaction was stopped by the addition of $6 \mu \mathrm{l}$ of $25 \mathrm{mM}$ EDTA and incubated for another 5 minutes, then phenol:chloroform extracted and the DNA was purified using AMPure XP beads (at ambient temperature as described above) and the libraries eluted in $10 \mu \mathrm{T}_{10} \mathrm{E}_{0.1}$ each. The remaining half of the circularised library was left untreated as a control.

\section{Library amplification and size-selection}

The libraries were amplified by PCR using indexed primers, corresponding to those used in the Illumina's TruSeq Small RNA Sample Prep Kit. One third of each of the PDDtreated and untreated library samples from the previous step were added as templates to PCR mixtures (Platinum ${ }^{\circ}$ Pfx DNA Polymerase supplemented with $1 \times$ buffer (Life Technologies), dNTPs, primers, and Extreme Thermophilic Single-Stranded Binding Protein (New England Biolabs)) and amplified through 15 thermocycles. Primers contained all the flanking sequences features necessary for sequencing on the intended platform, and different index sequences for each library. PCR products were run on a TBE DNA gel (Novex) and a band of $\sim 170-350$ bp (corresponding to insert sizes of 25-200 nt) was cut out, DNA from it was purified and analysed by on-chip electrophoresis using an Agilent Bioanalyzer. Libraries that were to be directly compared with each other (e.g. paired PDD-treated and untreated samples) were quantified and pooled together proportionally prior to size-selection on the gel. The indexed, size-selected libraries were pooled with other indexed size-selected libraries for sequencing from the $5^{\prime}$ ends (150 nt reads) on an Illumina HiSeq 2500 instrument by the Biomolecular Resource Facility, Australian National University.

\section{Sequence data analysis}

Raw reads were trimmed for low-quality positions (Phred $>28$ ) using fastq_quality_trimmer and adapter sequences and poly $(\mathrm{A})$ tracts were then trimmed using fastx_clipper, both from the fastx tools suite. Reads 
shorter than 11 nt after trimming were discarded. Reads were initially mapped unidirectionally using BowTie2 [16] (default settings) against processed rRNA sequences, and the unmapped reads were then mapped against other non-coding RNAs including rRNA precursors, snoRNAs and tRNAs. Finally, the remaining unmapped reads were mapped unidirectionally against mRNAs (all predicted spliced ORFs). Reference sequences were from the S288C reference genome (assembly R64-1-1, Saccharomyces Genome Database [15]). Read densities and counts per transcript were collated using custom Perl and $\mathrm{R}$ scripts. Reads were first divided into size ranges, and the density of the $5^{\prime}$ ends of reads mapping to rRNA was calculated as a moving average (window size $9 \mathrm{nt}$ ) from PDD-treated and untreated libraries. Read 5' density from PDD-treated libraries was plotted as a percentage of that from untreated libraries after normalizing for library loading using the total number of mRNA-mapped reads within the relevant insert size-group. Depletion efficiency was calculated from the minima of this value after averaging across all functional $18 \mathrm{~S}$ and $25 \mathrm{~S}$ probes.

GC content of libraries was calculated for all reads mapping to mRNAs from each library. PCR duplication rates were determined by analysing the number of reads starting at exactly the same position. Coverage was calculated as the number of reads divided by the number of available positions (estimated as the spliced ORF length minus the median read length) on each ORF. Simulated reshuffling of read 5' ends was performed repeatedly over the available positions in each ORF and the number of exact 5 ' read end overlaps was tallied and compared to the numbers from the real data (Additional file 2: Figure S2). At least 100 simulations were performed for ORFs with $>10$ reads, and at least 500 each for ORFs with $2-10$ reads.

\section{qPCR}

qPCR was performed using Quantifast qPCR premix (Qiagen) as per manufacturer's instructions with primers as listed in (Additional file 1: Table S1) at $0.5 \mu \mathrm{M}$ final concentration. Serial dilutions of control cDNA were used to test the PCR efficiency, which was $>90 \%$ for all primers. $10 \mu \mathrm{l}$ reactions were generated in 384-well plates (3 technical replicates per condition) and qPCR performed on a QuantStudio 12 K Flex (Invitrogen).

\section{Results}

In developing PDD we aimed for a method that would avoid manipulations at the RNA level and use unmodified DNA oligonucleotide probes to allow for cost savings as well as facile customisation. These criteria were met by deploying probe-mediated DSN cleavage at the stage of single-stranded adapter-flanked cDNA, common to several RNA-seq library preparation methods, including the split adapter ligation approach used here (Figure 1) [2]. A single cut introduced anywhere in a library molecule will prevent its amplification via the adapter sequences at subsequent stages. This strategy also avoids inadvertent cleavage of adapter sequences, as there is no complementary strand with which they could form dsDNA. DSN operates efficiently at higher temperatures and requires at least ten perfectly complementary base pairs to cut, thus it has better mismatch discrimination than RNase $\mathrm{H}$, especially given the higher $\mathrm{T}_{\mathrm{m}}$ of RNA:DNA compared to DNA: DNA hybrids. That PDD is performed downstream of adapter ligation furthermore eliminates any risk of new adapter-insert junctions being created through off-target cutting.

\section{Proof of concept}

To test the feasibility of the PDD strategy, we simulated the generation of a RNA-seq library from degraded RNA by using RNase I-treated S. cerevisiae cytoplasmic lysate as starting material for the split-adapter library preparation method [2]. This method, which has been extensively characterized elsewhere $[17,18]$ and is now also implemented as a commercial kit, was chosen due to its even coverage and low tendency for insert bias. It generates a single-stranded intermediate antisense cDNA construct flanked by $5^{\prime}$ and $3^{\prime}$ adapters in a circular molecule (Figure 1). We mixed the library intermediate

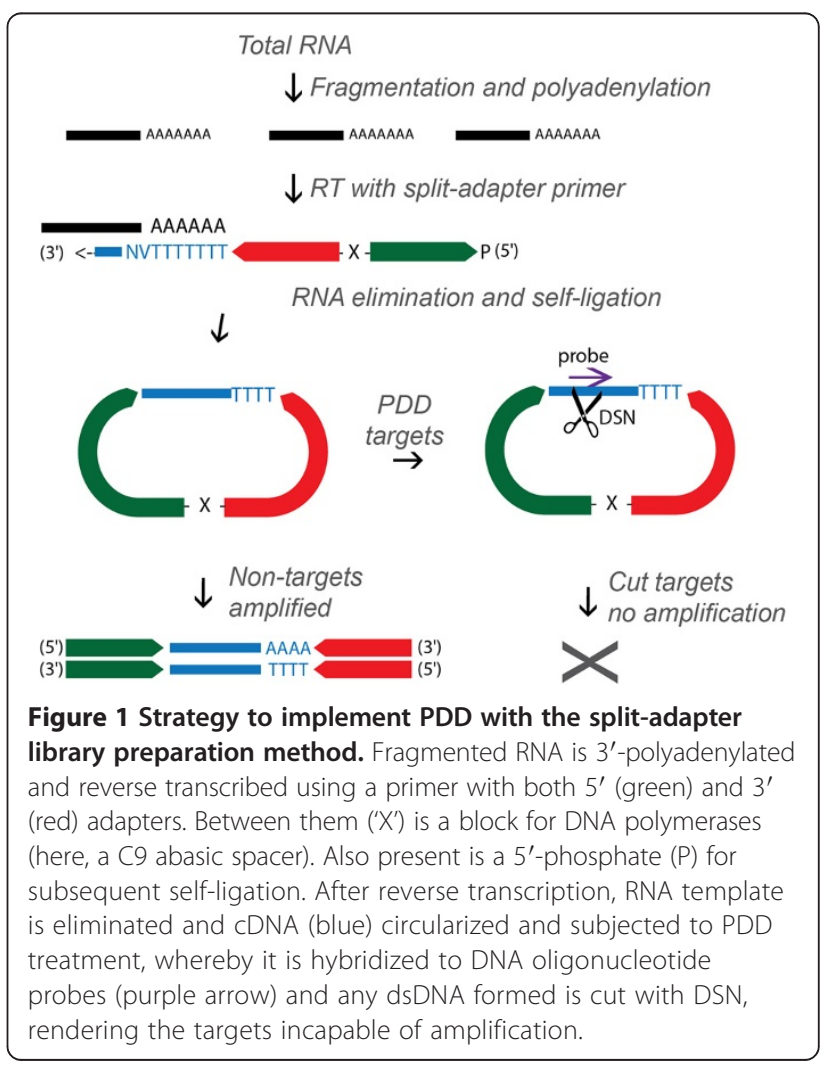


with four oligonucleotide probes corresponding to rRNA sense sequences (Figure 2A, top), denatured and cooled to $48^{\circ} \mathrm{C}$, whereupon DSN was added to one half of the mixture and the other mock incubated. After library amplification, qPCR was performed for several rRNA amplicons as well as mRNA sequences for normalisation purposes. This showed that the abundance of the two directly targeted sequences (rRNA-A and rRNA-B) had decreased by approximately two orders of magnitude in the DSN-treated library, while another qPCR amplicon (rRNA-C), 100 nt away from two flanking probe-targeted sequences, still decreased by about 5 -fold (Figure 2A, bottom).

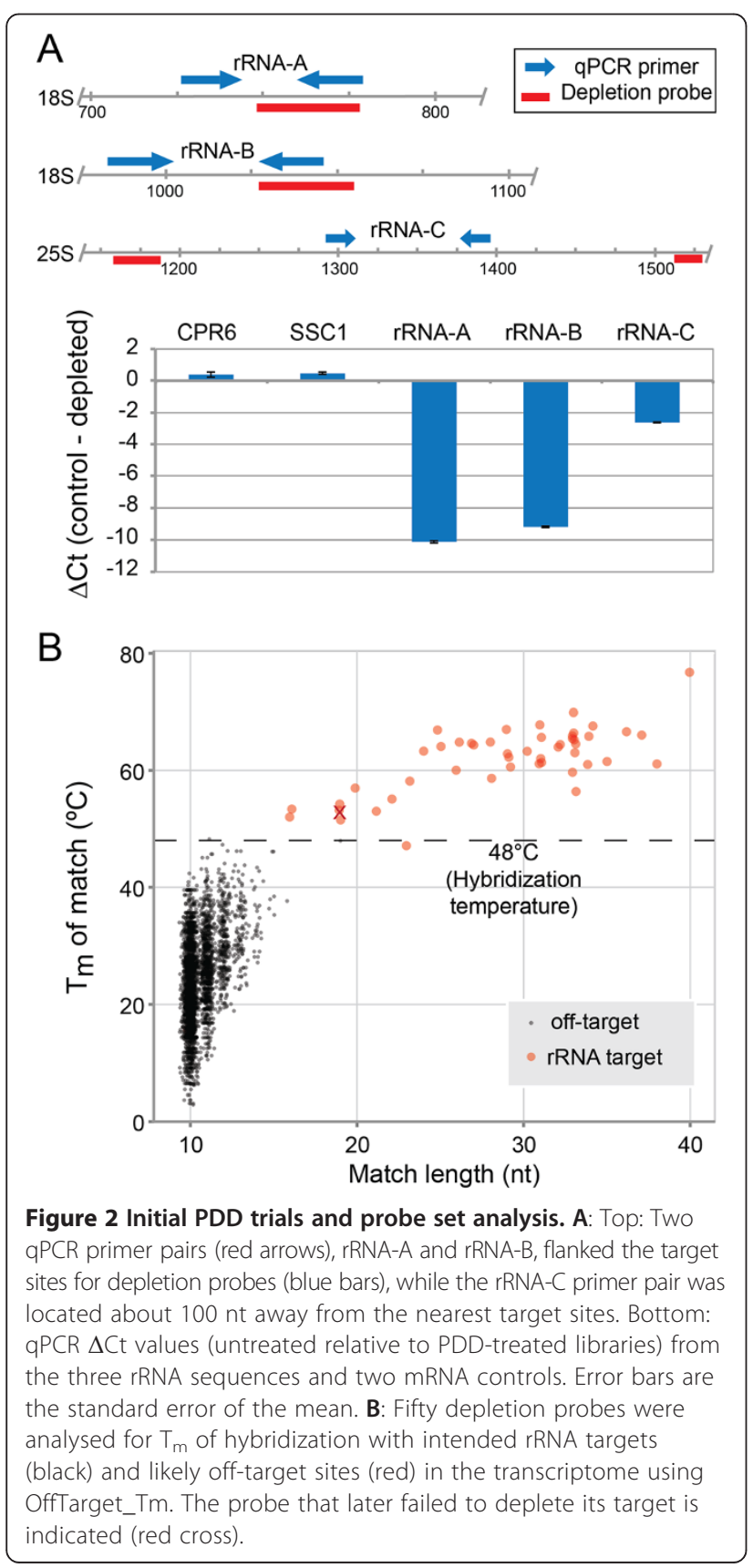

\section{Probe design}

Following these encouraging findings, we designed more sense-strand DNA oligonucleotide probes to target rRNA fragments that tend to dominate ribosome profiling libraries to reach a total of 24 each, against $18 \mathrm{~S}$ and $25 \mathrm{~S}$ rRNA, and one each, against 5.8S and 5S rRNA (see Additional file 1: Table S1). The probes had variable spacing across the rRNAs, allowing characterisation of individual probe efficacy and probe spacing requirements. We also generated a bioinformatics tool, OffTarget_Tm (Additional file 3), to evaluate probe sets. OffTarget_Tm performs in silico annealing of probes to the target RNAs and other transcriptome sequences in order to identify potential unintentional off-targets and determine the optimum hybridization temperature for PDD. Results with our probe set indicated excellent discrimination between intended targets and off-targets at our chosen hybridization temperature of $48^{\circ} \mathrm{C}$ (dashed line, Figure $2 \mathrm{~B}$ ).

\section{Application to RNA-seq libraries}

To demonstrate the performance of PDD in full-scale RNA-seq experiments, we generated two high-complexity RNA-seq libraries by the split adapter method, this time using $\mathrm{Mg}^{+2}$-mediated fragmentation of purified S. cerevisiae RNA. One library was made from highly fragmented total RNA (8 minute treatment). The other library was made from a moderately fragmented (6 minute treatment) mixture of total RNA spiked with $20 \%$ of RNA that had been enriched for mRNA using the RiboMinus ${ }^{\text {TM }}$ kit. The purpose of the first library was to assess PDD efficacy in a typical application. The goal with the second library was to have better mRNA representation (particularly for the untreated control sample) to accurately assess any offtarget effects of PDD. We also modified the published split adapter ligation protocol (see Methods) to include selection of cDNA:RNA duplexes away from unextended reverse-transcription primer (which can cause adapter dimers to form in subsequent steps), while bypassing several gel-electrophoresis purification steps.

Each cDNA library intermediate was split into equal aliquots and either PDD treated or left untreated. The four samples were then PCR-amplified, size-selected for an insert size of $\sim 20-200 \mathrm{nt}$ and the first $150 \mathrm{nt}$ sequenced from the $5^{\prime}$ end in a multiplexed run on an Illumina HiSeq 2500 instrument.

\section{Efficient depletion of rRNA}

We first focussed on the rRNA-mapped reads in the highly fragmented total RNA libraries. Densities of the $5^{\prime}$ ends of rRNA-mapped reads were calculated across all rRNAs and normalised to mRNA-mapped reads to correct for library loading. We then calculated 5 ' read density in the PDD-treated sample as a percentage of that in the untreated control, to characterise zones of 
depletion at each probe-targeted region. Conceptually, a steep drop in $5^{\prime}$ read density should be seen on the right flank of the zones, starting beyond ten nucleotides upstream from the location of probe $3^{\prime}$ ends, as a direct consequence of DSN's minimum requirement of ten consecutive matching base pairs for degradation [19]. Conversely, position and slope of the left flanks of the depletion zones should be determined by the distribution of insert sizes in the libraries (see schematic in
Additional file 2: Figure S1A). To ascertain these patterns in our experiment we performed several in silico size selections, simulating libraries of different insert size ranges, and plotted their relative $5^{\prime}$ read densities across rRNAs. Figure 3A shows $5^{\prime}$ read densities in PDD-treated libraries, as a percentage of untreated, along the entire $18 \mathrm{~S}$ rRNA. It is apparent that each probe created a zone of depletion that grew wider with increasing insert size. Overall, we saw efficient and consistent depletion for 49 of

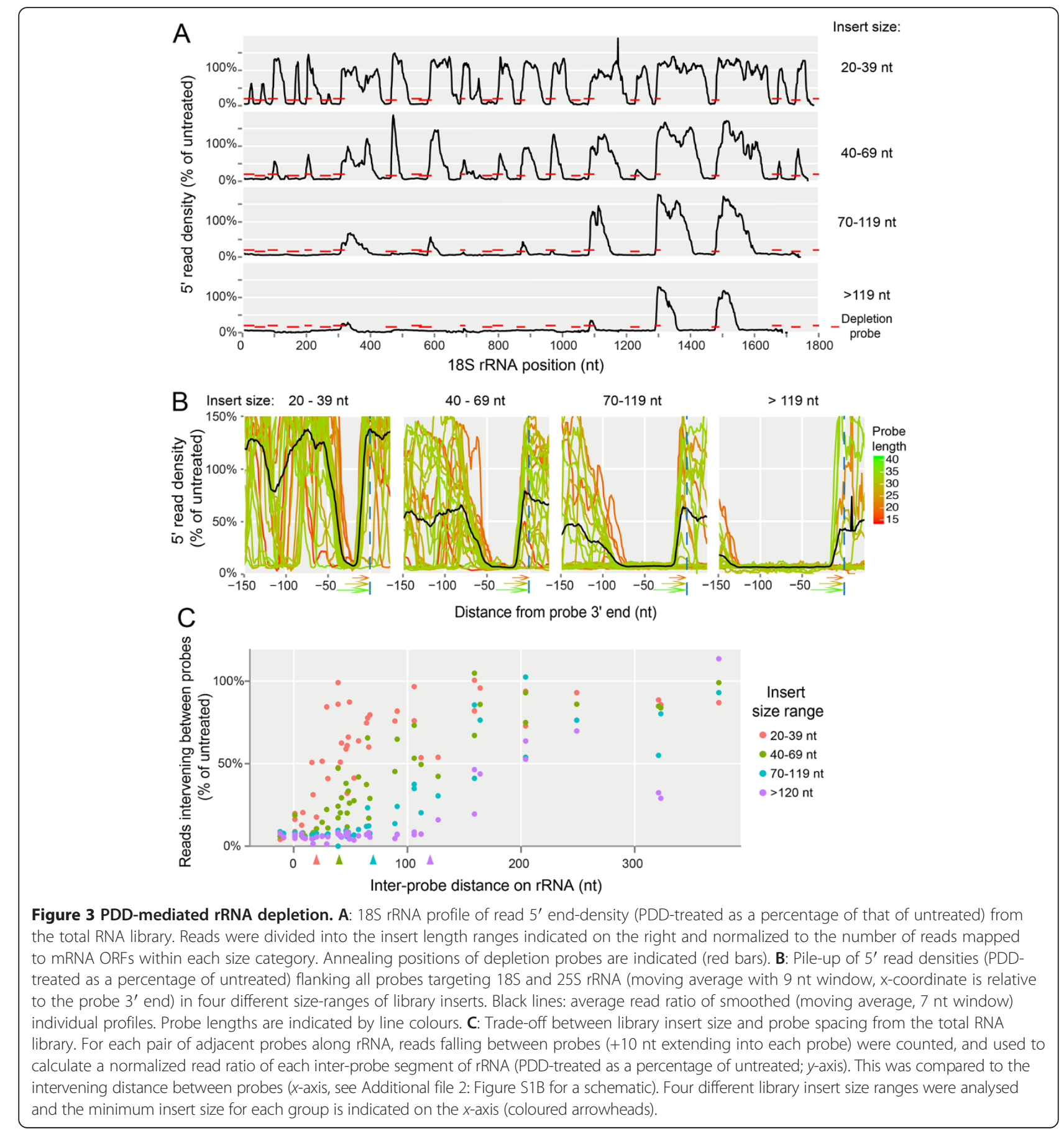


the 50 probes. The reason for failure of one probe (against $25 \mathrm{~S}$ rRNA, position 1512) is unclear, although it was shorter than most of the others (see red ' $x$ ' in Figure 2B), thus it may be prudent to design probes of $>22$ nt length. Figure 3B displays all probe-targeted regions in $18 \mathrm{~S}$ and $25 \mathrm{~S}$ rRNA (except that of the failed $25 \mathrm{~S}$ probe), aligned according to the 3 ' end of each probe, to assess depletion zone topology for different library insert size ranges. Consistent across all probes we saw the expected steep drop in read density beyond ten nucleotides upstream of probe 3 ' ends. Coverage recovered further upstream as a function of insert length cutoffs, with longer library inserts yielding larger depletion zones. We next explored the relationship between library insert size, inter-probe distance and read depletion. PDD should achieve continuous rRNA depletion for inter-probe distances that are at least $20 \mathrm{nt}$ shorter than the minimum insert size of the library. This would allow every rRNA insert to be targeted by at least one probe (Additional file 2: Figure S1B). The results obtained from tallying reads falling between probes (Figure 3C) fully confirmed these expectations. For each insert size-range analysed, the relative depletion of inter-probe reads was greater for more closely spaced probes, approaching full efficiency as inter-probe distance dropped below the minimum insert size cutoff minus $20 \mathrm{nt}$. Assessing all inter-probe regions below this distance requirement further allowed us to calculate an average depletion efficiency of $\sim 94 \%$ for PDD in its current configuration.

\section{Absence of bias in retained transcriptome coverage}

We assessed several quality parameters in all four sequencing library datasets (Additional file 1: Table S2) and found no notable change with DSN treatment. The GC content of mRNA reads was not appreciably affected by $\mathrm{PDD}(<2 \%$ difference), unlike previously reported with $\mathrm{C}_{0} \mathrm{~T}$ hybridization [20]. Focussing on ORF-mapped reads in the mRNA-enriched libraries, $<2 \%$ of reads were PCR duplicates and little fragment bias was detected when comparing the observed read distribution to that produced by random reshuffling of reads, with or without PDD treatment (Additional file 2: Figure S2). These libraries again displayed the expected rRNA depletion pattern in the PDD-treated sample (data not shown), and were further processed to assess mRNA representation (Figure 4). This revealed high concordance between libraries and, importantly, no systematic bias as a result of the DSN treatment, even for mRNAs carrying potential off-target sites for the depletion probes (red dots) as predicted by OffTarget_Tm.

\section{Discussion}

In this study we characterised PDD as an efficient, accurate and flexible method for the depletion of problematic

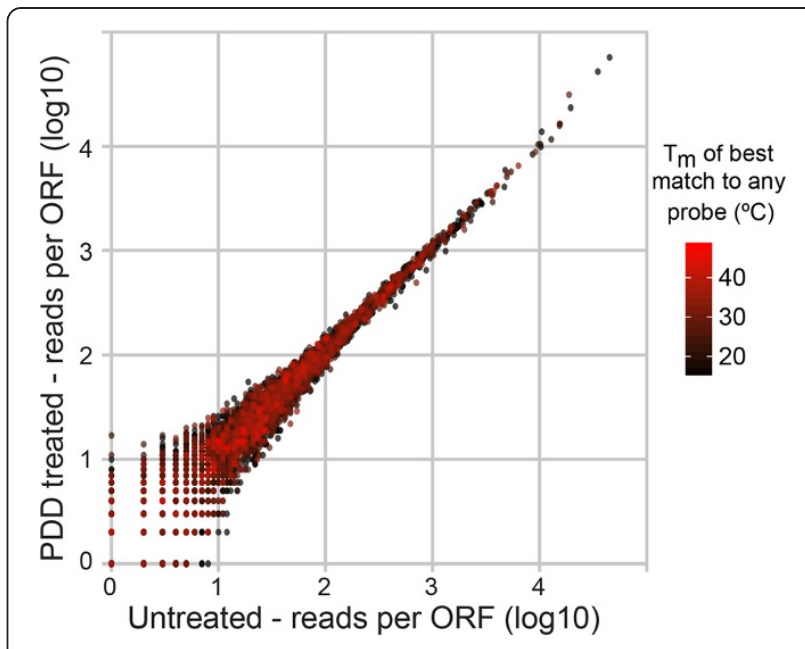

Figure 4 Comparison of read counts for mRNAs from both PDD treated and untreated libraries, derived from the $20 \%$ RiboMinus $^{\text {TM }}$ RNA spiked sample. Calculated $T_{m}$ of the best off-target match within an ORF to any of the probes is indicated by the intensity of red colour.

sequences from RNA-seq libraries. In applying it to $S$. cerevisiae rRNA we showed that PDD performance is reliable and predictable as a function of the well characterised enzymatic properties of DSN, probe design (using our OffTarget_Tm tool) and spacing, as well as library insert size distribution. When starting with intact total RNA samples there is no need for contiguous probe coverage of the target, rather a probe spacing of 20 nt less than the minimum anticipated library insert size, or closer, will suffice to obtain even and continuous depletion. rRNA depletion by PDD in its present configuration is slightly less efficient ( $\sim 94 \%)$ than that reported for the RNase $\mathrm{H}$ method or commercial Ribo-Zero $^{\text {тм }} /$ RiboMinus $^{\text {тм }}$ kits (reportedly $>99 \%$ $[7,8])$. Offsetting this are other advantages of PDD such as its high stringency/lack of bias and avoidance of nuclease exposure prior to linker ligation. The relatively sparse probe spacing required further increases flexibility of probe design so that off-targets can be avoided.

Given the low cost of unmodified DNA oligonucleotides, PDD is an affordable solution for depletion of rRNA in species for which off-the-shelf commercial kits are not available. Similarly, PDD might be a cost-effective option when sequencing large sample sets derived from standard organisms. Its faithful preservation of fragment ends makes it highly suitable for applications that rely heavily on accurate mapping of fragment ends, such as ribosome profiling, CLIP-seq and related approaches, mapping of transcript extremities, and nucleotide-resolution mapping of base modification in RNA (e.g. by aniline-mediated RNA cleavage) [21,22]. As PDD operates at the cDNA level it could also be applied to remediate pre-existing libraries containing unacceptable levels of unwanted sequences (e.g. rRNA after failed 
depletion attempts with the source RNA sample) or to remove "adapter dimers" (by targeting adapter-adapter junctions).

We implemented PDD in combination with the split adapter method, however, it should be applicable to most directional RNA-seq library preparation methods, with some modifications, if it is performed after linker ligation and reverse transcription but before amplification (although some precautionary measures, e.g. linker decoys or modified linkers, may be necessary if one or both linkers are double-stranded). We have tested $1 \times$ DSN buffer for hybridization and DSN digestion. While buffers that are similar in terms of ionic strength and $\mathrm{pH}$ should also permit PDD, any free divalent cations other than $\mathrm{Mg}^{+2}$ (e.g. $\mathrm{Mn}^{+2}$ such as is used in the CircLigase ${ }^{\mathrm{Tm}}$ buffer) should be removed or chelated with equimolar EDTA (which binds Mn +2 preferentially to $\mathrm{Mg}^{+2}$ ) as they are known to alter DSN activity [23] and interfere with fidelity of a related dsDNA nuclease [24]. Note that it is important to eliminate the template RNA prior to PDD as DSN can also degrade the DNA strand of RNA:DNA hybrids longer than 15 nt [23]. Conversely, this property of DSN might even be harnessed productively, by using purified fragmented rRNA instead of oligonucleotides to direct DSN cleavage.

Additional potential applications and variations of the PDD procedure are numerous. Identification of minor organisms present in mixed environmental samples (e.g. for microbial transcriptomics) might also be aided by PDD, through selective depletion of conserved rRNA loci, allowing deeper sequencing of more variable rRNA regions for discriminating between species. The rapid flexibility of PDD also allows unwanted sequences other than rRNA or adapter dimers to be readily targeted for a variety of applications. Highly abundant mRNAs in the target cell or tissue to be sequenced (e.g. globin mRNA from blood cells [25]) could be depleted. Capture-seq, a technique for focussed sequencing of rare transcripts [1], could be combined with PDD to deplete abundant transcript isoforms or contaminants, for example to find rare expressed antibody variants in immune cell populations responding to antigen. The mismatch-discrimination of DSN could potentially allow the enrichment of rare point mutation-containing sequences in other types of pooled samples [19], for example to analyse the deep mutational landscape of retrovirus in a patient's bloodstream.

\section{Conclusions}

We envisage that PDD will prove useful for ridding RNA-seq libraries of sequences that would otherwise dominate coverage. Key features of PDD are its relatively low cost, flexibility and accuracy, allowing it to be customised to a variety of source species and applications. In this way PDD will facilitate the ever increasing and diverse uses of RNA-seq in the modern life sciences.

\section{Data availability}

Sequencing data is available at the NCBI SRA database (www.ncbi.nlm.nih.gov/sra) under project accession number: SRP041813.

\section{Additional files}

Additional file 1: Table S1. Is a list of oligonucleotides used in this study. Table S2. Is a summary of diagnostic statistics for sequencing quality from the sequencing run.

Additional file 2: Figure S1A and S1B. Are schematic diagrams describing expected $5^{\prime}$ read densities after PDD treatment, and Figure S2. shows an analysis of PCR-duplication in the sequencing data.

Additional file 3: Contains a Perl script, Offtarget_tm.pl that was used to estimate matches between probes and transcripts in an annotated genome.

\section{Competing interests}

The authors declare that they have no competing interests.

\section{Authors' contributions}

SKA and TP conceived and designed the experiments. SKA and NES performed the experiments. SKA analyzed the data. SKA and TP wrote the paper. All authors read and approved the manuscript.

\section{Acknowledgements}

This work was supported by an Australian Research Council Discovery Grant (DP1300101928) and a NHMRC Senior Research Fellowship (514904) awarded to TP. NES was supported by a Go8 European Fellowship. We acknowledge technical support from the Australian Cancer Research Foundation Biomolecular Resource Facility.

\section{Author details}

${ }^{1}$ Genome Biology Department, The John Curtin School of Medical Research (JCSMR), The Australian National University, Acton, Canberra, Australian Capital Territory, Australia. ${ }^{2}$ Present address - Moscow Regional State Institute of Humanities and Social Studies, Ministry of Education of Moscow Region, Kolomna, Moscow Region, Russia.

Received: 18 March 2014 Accepted: 16 May 2014

Published: 26 May 2014

\section{References}

1. Mercer TR, Gerhardt DJ, Dinger ME, Crawford J, Trapnell C, Jeddeloh JA, Mattick JS, Rinn JL: Targeted RNA sequencing reveals the deep complexity of the human transcriptome. Nat Biotechnol 2012, 30:99-104.

2. Ingolia NT, Ghaemmaghami S, Newman JRS, Weissman JS: Genome-wide analysis in vivo of translation with nucleotide resolution using ribosome profiling. Science 2009, 324:218-223.

3. Ingolia NT, Brar GA, Rouskin S, McGeachy AM, Weissman JS: The ribosome profiling strategy for monitoring translation in vivo by deep sequencing of ribosome-protected mRNA fragments. Nat Protoc 2012, 7:1534-1550.

4. Ule J, Jensen K, Mele A, Darnell RB: CLIP: a method for identifying protein-RNA interaction sites in living cells. Methods 2005, 37:376-386.

5. Licatalosi DD, Mele A, Fak JJ, Ule J, Kayikci M, Chi SW, Clark TA, Schweitzer AC, Blume JE, Wang X, Darnell JC, Darnell RB: HITS-CLIP yields genome-wide insights into brain alternative RNA processing. Nature 2008, 456:464-469.

6. Murigneux V, Saulière J, Roest Crollius H, Le Hir H: Transcriptome-wide identification of RNA binding sites by CLIP-seq. Methods 2013, 63:32-40.

7. Adiconis X, Borges-Rivera D, Satija R, DeLuca DS, Busby MA, Berlin AM, Sivachenko A, Thompson DA, Wysoker A, Fennell T, Gnirke A, Pochet N, Regev A, Levin JZ: Comparative analysis of RNA sequencing methods for degraded or low-input samples. Nat Methods 2013, 10:623-629.

8. Morlan JD, Qu K, Sinicropi DV: Selective depletion of rRNA enables whole transcriptome profiling of archival fixed tissue. PLOS ONE 2012, 7:e42882.

9. Casey J, Davidson N: Rates of formation and thermal stabilities of RNA: DNA and DNA: DNA duplexes at high concentrations of formamide. Nucleic Acids Res 1977, 4:1539-1552. 
10. Ko MSH: An "equalized cDNA library" by the reassociation of short double-stranded cDNAs. Nucleic Acids Res 1990, 18:5705-5711.

11. Yi H, Cho Y-J, Won S, Lee J-E, Jin Yu H, Kim S, Schroth GP, Luo S, Chun J: Duplex-specific nuclease efficiently removes rRNA for prokaryotic RNA-seq. Nucleic Acids Res 2011, 39:e140.

12. VanderNoot VA, Langevin SA, Solberg OD, Lane PD, Curtis DJ, Bent ZW, Williams KP, Patel KD, Schoeniger JS, Branda SS, Lane TW: CDNA normalization by hydroxyapatite chromatography to enrich transcriptome diversity in RNA-seq applications. BioTech 2012, 53:373-380.

13. Bogdanova EA, Shagina IA, Mudrik E, Ivanov I, Amon P, Vagner LL, Lukyanov SA, Shagin DA: DSN depletion is a simple method to remove selected transcripts from cDNA populations. Mol Biotechnol 2009, 41:247-253.

14. Allawi HT, SantaLucia J: Thermodynamics and NMR of Internal G · T Mismatches in DNA. Biochemistry 1997, 36:10581-10594.

15. Engel SR, Dietrich FS, Fisk DG, Binkley G, Balakrishnan R, Costanzo MC, Dwight SS, Hitz BC, Karra K, Nash RS, Weng S, Wong ED, Lloyd P, Skrzypek MS, Miyasato SR, Simison M, Cherry JM: The reference genome sequence of saccharomyces cerevisiae: then and now. G3 (Bethesda) 2014, 4:389-398.

16. Langmead B, Salzberg SL: Fast gapped-read alignment with Bowtie 2. Nat Methods 2012, 9:357-359.

17. Lamm AT, Stadler MR, Zhang H, Gent Jl, Fire AZ: Multimodal RNA-seq using single-strand, double-strand, and CircLigase-based capture yields a refined and extended description of the $\mathrm{C}$. elegans transcriptome. Genome Res 2011, 21:265-275.

18. Levin JZ, Yassour M, Adiconis X, Nusbaum C, Thompson DA, Friedman N, Gnirke A, Regev A: Comprehensive comparative analysis of strand-specific RNA sequencing methods. Nat Methods 2010, 7:709-715.

19. Shagin DA: A novel method for SNP detection using a new duplex-specific nuclease from crab hepatopancreas. Genome Res 2002, 12:1935-1942.

20. Giannoukos G, Ciulla DM, Huang K, Haas BJ, Izard J, Levin JZ, Livny J, Earl AM, Gevers D, Ward DV, Nusbaum C, Birren BW, Gnirke A: Efficient and robust RNA-seq process for cultured bacteria and complex community transcriptomes. Genome Biol 2012, 13:r23.

21. Behm Ansmant I, Helm M, Motorin Y: Use of specific chemical reagents for detection of modified nucleotides in RNA. J Nucleic Acids 2011, 2011:1-17.

22. Squires JE, Patel HR, Nousch M, Sibbritt T, Humphreys DT, Parker BJ, Suter CM, Preiss T: Widespread occurrence of 5-methylcytosine in human coding and non-coding RNA. Nucleic Acids Res 2012, 40:5023-5033.

23. Anisimova VE, Rebrikov DV, Shagin DA, Kozhemyako VB, Menzorova NI, Staroverov DB, Ziganshin R, Vagner LL, Rasskazov VA, Lukyanov SA, Shcheglov AS: Isolation, characterization and molecular cloning of Duplex-Specific Nuclease from the hepatopancreas of the Kamchatka crab. BMC Biochem 2008, 9:14.

24. Nilsen IW, Øverbø K, Jensen Havdalen L, Elde M, Gjellesvik DR, Lanes O: The enzyme and the CDNA sequence of a thermolabile and double-strand specific DNase from Northern Shrimps (Pandalus borealis). PLOS ONE 2010, 5:e10295.

25. Mastrokolias A, den JT D, van Ommen GB, Hoen PAC 't, van Roon-Mom WMC: Increased sensitivity of next generation sequencing-based expression profiling after globin reduction in human blood RNA. BMC Genomics 2012, 13:28

doi:10.1186/1471-2164-15-401

Cite this article as: Archer et al:: Selective and flexible depletion of problematic sequences from RNA-seq libraries at the CDNA stage. BMC Genomics 2014 15:401.

\section{Submit your next manuscript to BioMed Central and take full advantage of:}

- Convenient online submission

- Thorough peer review

- No space constraints or color figure charges

- Immediate publication on acceptance

- Inclusion in PubMed, CAS, Scopus and Google Scholar

- Research which is freely available for redistribution 